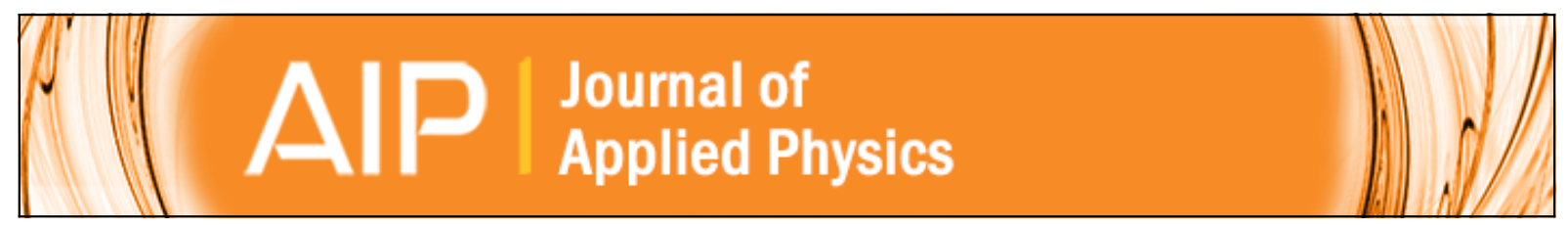

\title{
Ferromagnetism in chromium-doped reduced-rutile titanium dioxide thin films
}

Zhenjun Wang, Jinke Tang, Hongguo Zhang, Vladimir Golub, Leonard Spinu, and Le Duc Tung

Citation: Journal of Applied Physics 95, 7381 (2004); doi: 10.1063/1.1667806

View online: http://dx.doi.org/10.1063/1.1667806

View Table of Contents: http://scitation.aip.org/content/aip/journal/jap/95/11?ver=pdfcov

Published by the AIP Publishing

\section{Articles you may be interested in}

Room temperature ferromagnetism in transition metal ( $\mathrm{V}, \mathrm{Cr}, \mathrm{Ti})$ doped In 203

J. Appl. Phys. 101, 09N513 (2007); 10.1063/1.2712018

Transport properties in chromium-doped Ti 203 thin films

J. Appl. Phys. 97, 10D319 (2005); 10.1063/1.1852855

Ferromagnetic $\mathrm{Cr}$-doped $\mathrm{ZnO}$ for spin electronics via magnetron sputtering

J. Appl. Phys. 97, 10D310 (2005); 10.1063/1.1847914

Room-temperature ferromagnetism in manganese doped reduced rutile titanium dioxide thin films

J. Appl. Phys. 95, 7384 (2004); 10.1063/1.1667858

Ferromagnetism and transport properties of Fe-doped reduced-rutile TiO $2-\delta$ thin films

J. Appl. Phys. 93, 7870 (2003); 10.1063/1.1556122

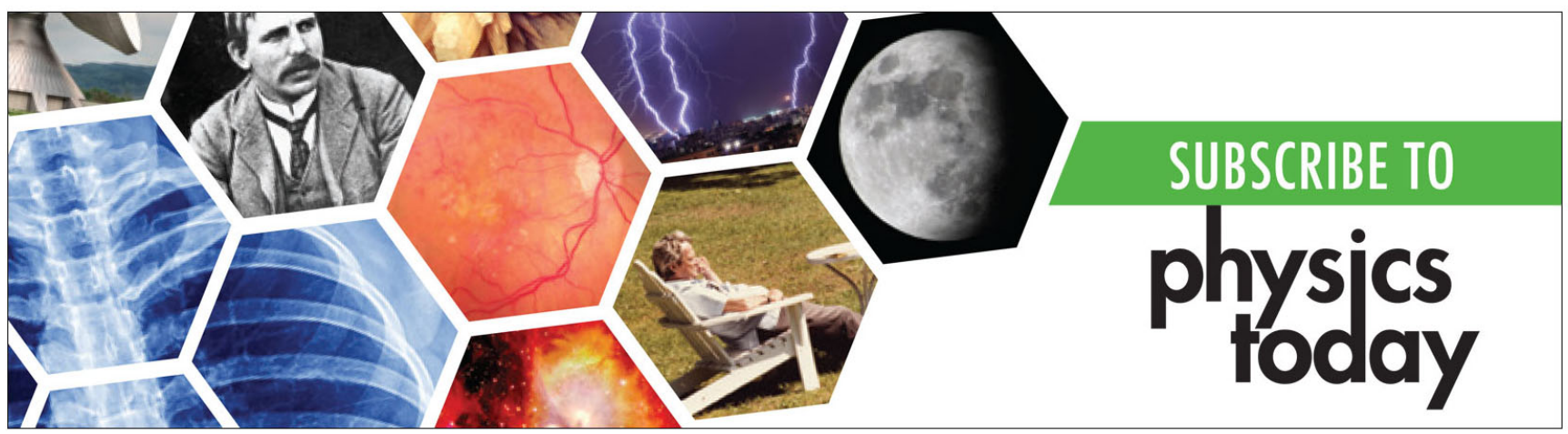




\title{
Ferromagnetism in chromium-doped reduced-rutile titanium dioxide thin films
}

\author{
Zhenjun Wang and Jinke Tang ${ }^{\text {a) }}$ \\ Department of Physics, University of New Orleans, New Orleans, Louisiana 70148 \\ Hongguo Zhang, Vladimir Golub, and Leonard Spinu \\ Advanced Materials Research Institute, University of New Orleans, New Orleans, Louisiana 70148 \\ Le Duc Tung \\ Department of Physics, University of Warwick, Coventry CV4 7AL, United Kingdom
}

(Presented on 9 January 2004)

Cr-doped reduced-rutile $\mathrm{TiO}_{2}$ thin films were grown on $R$-plane sapphire substrates by pulsed-laser deposition. X-ray diffraction and transmission electron microscopy results indicate that the films are single phase and reduced-rutile type. Superconducting quantum interference device magnetometer measurements show the films are ferromagnetic up to $400 \mathrm{~K}$. A large magnetic moment of $2.9 \mu_{\mathrm{B}}$ per $\mathrm{Cr}$ atom is found for $6 \% \mathrm{Cr}$-doped reduced films at room temperature, and the saturation magnetization of the films decreases with increasing $\mathrm{Cr}$ doping. The temperature dependence of the resistivity shows semiconducting behavior. (c) 2004 American Institute of Physics.

[DOI: $10.1063 / 1.1667806]$

\section{INTRODUCTION}

Because of the combined magnetic and transport properties in dilute magnetic semiconductors and the potential technological applications, a growing effort is directed toward studies of magnetic semiconductors with practical ordering temperature. ${ }^{1,2}$ Recently, above room temperature, ferromagnetism has been reported ${ }^{3,4}$ in Fe-doped reduced-rutile $\mathrm{TiO}_{2}$ and in Co-doped $\mathrm{SnO}_{2}$ of rutile structure. ${ }^{5}$ Rutile $\mathrm{TiO}_{2}$ crystallizes in a tetragonal lattice and is easily reduced by forming oxygen vacancies or titanium interstitials, and the reduced rutile forms a homologous series of compounds $\mathrm{Ti}_{n} \mathrm{O}_{2 n-1}$ with triclinic structure. Although the earlier investigations of the phase relations within the composition range $\mathrm{MO}_{1.75-2.0}(\mathrm{M}=\mathrm{Ti}, \mathrm{Cr}, \ldots)$ of the titanium-chromium-oxygen system revealed the existence of analogous series of oxides $\mathrm{Ti}_{n-2} \mathrm{Cr}_{2} \mathrm{O}_{2 n-1}(6 \leqslant n \leqslant 9)$ with triclinic structure, ${ }^{6-9}$ ferromagnetic properties at RT have not been reported in titanium-chromium-oxygen system.

In this work, following the study of Fe-doped reducedrutile $\mathrm{TiO}_{2}$ films, ${ }^{3,4}$ we have investigated the magnetic and transport properties of $\mathrm{Cr}$-doped reduced-rutile $\mathrm{TiO}_{2}$ thin films.

\section{EXPERIMENT}

$\mathrm{Cr}_{x} \mathrm{Ti}_{1-x} \mathrm{O}_{2-\delta}(x=0.06,0.08,0.12,0.14)$ thin films were grown on $\alpha-\mathrm{Al}_{2} \mathrm{O}_{3}(012)$ substrates by pulsed-laser deposition (PLD). $\mathrm{Cr}_{x} \mathrm{Ti}_{1-x} \mathrm{O}_{2-\delta}$ targets were prepared using standard ceramic techniques. The films were prepared in vacuum at a substrate temperature of $800-1000 \mathrm{~K}$. The pressure during deposition was $2 \times 10^{-6}$ Torr. The pulsed excimer laser uses $\operatorname{KrF}(\lambda=248 \mathrm{~nm})$ and produces a laser beam of intensity of $1-2 \mathrm{~J} / \mathrm{cm}^{2}$ and repetition rate of $4 \mathrm{~Hz}$. The

a)Electronic mail: jtang@uno.edu deposition rate is between 0.2 and $0.4 \AA / \mathrm{s}$, and the film thickness varies from 50 to $100 \mathrm{~nm}$. The $\mathrm{Cr}$ concentrations of the films were measured with energy dispersive $\mathrm{x}$-ray analysis in TE mode, and they were consistent with those of the targets. The crystalline structure was investigated by $\mathrm{x}$-ray diffraction (XRD) with $\mathrm{Cu} \mathrm{K}_{\alpha}$ radiation and transmission electron microscopy (TEM). The magnetic properties were studied with a superconducting quantum interference device (SQUID) magnetometer. The transport properties were measured with a physical property measurement system from Quantum Design.

\section{RESULTS AND DISCUSSION}

Figure 1 shows the XRD pattern for a $\mathrm{Cr}_{0.06} \mathrm{Ti}_{0.94} \mathrm{O}_{2-\delta}$ film grown on $R$-plane $\alpha-\mathrm{Al}_{2} \mathrm{O}_{3}$ (012). The film is single phase and of reduced-rutile type with (202) plane, referred to the stoichiometric rutile tetragonal cell, parallel to the film

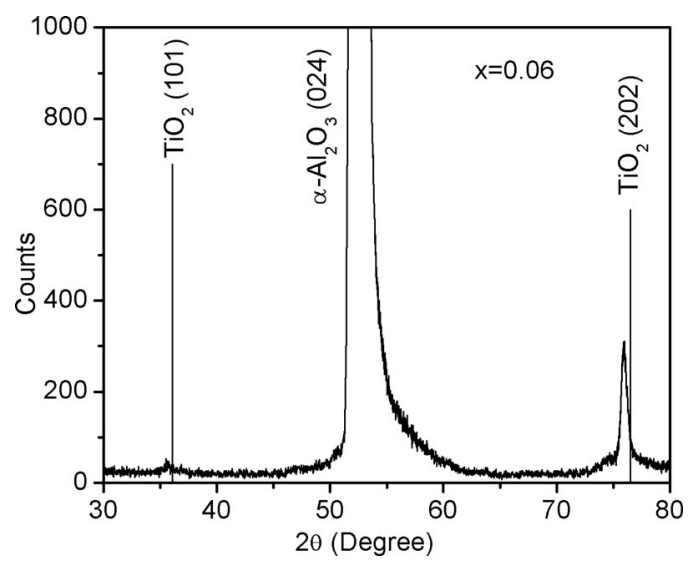

FIG. 1. XRD pattern of a $\mathrm{Cr}_{0.06} \mathrm{Ti}_{0.94} \mathrm{O}_{2-\delta}$ film grown on $\alpha-\mathrm{Al}_{2} \mathrm{O}_{3}$ (012) substrate showing reduced-rutile structure. 


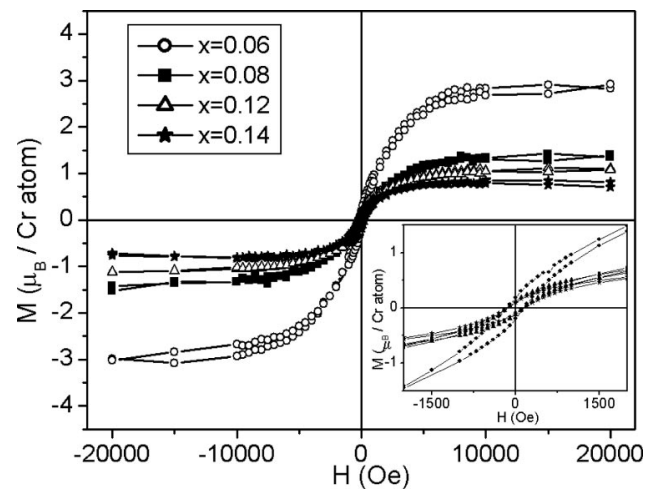

FIG. 2. Magnetic hysteresis loops $\mathrm{Cr}_{x} \mathrm{Ti}_{1-x} \mathrm{O}_{2-\delta}(x=0.06,0.08,0.12,0.14)$ films measured with a SQUID at RT. The inset shows the low-field region of the hysteresis loop for the four films.

plane. The two vertical lines show (101) and (202) reflections of the stoichiometric rutile. The peak at the center of the scans is that of the substrate $\alpha-\mathrm{Al}_{2} \mathrm{O}_{3}$ (024). The (202) peaks are shifted toward lower $2 \theta$ angles. The (101) peak is absent due to substantial reduction that forms the Magnéli shear plane. Similar XRD patterns were obtained for all films with different $\mathrm{Cr}$ content. The XRD results are similar to that of the Fe-doped reduced-rutle $\mathrm{TiO}_{2} \cdot{ }^{3,4} \mathrm{XRD}$ and TEM observation indicate no sign of the existence of any impurity phase up to $x=0.14$ in this study. In fact, much higher $\mathrm{Cr}$ content can be sustained in the analogous series of oxides $\mathrm{Ti}_{n-2} \mathrm{Cr}_{2} \mathrm{O}_{2 n-1}(6 \leqslant n \leqslant 9)$ with triclinic structure. ${ }^{8,9}$ The TEM observation showed the films are epitaxially grown.

In Fig. 2, we show magnetic hysteresis loops at RT for $\mathrm{Cr}_{0.06} \mathrm{Ti}_{0.94} \mathrm{O}_{2-\delta}, \quad \mathrm{Cr}_{0.08} \mathrm{Ti}_{0.92} \mathrm{O}_{2-\delta}, \quad \mathrm{Cr}_{0.12} \mathrm{Ti}_{0.88} \mathrm{O}_{2-\delta}$ and $\mathrm{Cr}_{0.14} \mathrm{Ti}_{0.86} \mathrm{O}_{2-\delta}$. The hysteresis loops show that all the films are ferromagnetic at RT. The coercivity of the films at RT is $149,134,140$, and 148 Oe for $x=0.06,0.08,0.12$, and 0.14 , respectively. The inset shows the low-field region of the hysteresis loops for the four films. The magnetic moment for the $\mathrm{Cr}_{0.06} \mathrm{Ti}_{0.94} \mathrm{O}_{2-\delta}$ film is about $2.9 \mu_{\mathrm{B}} / \mathrm{Cr}$, and the magnetic moment decreases with increasing $x$, as shown in Fig. 2 . This result indicates that, as the $\mathrm{Cr}$ concentration in the material increases, a decreasing fraction of their spins participate in the ferromagnetism.

Figure 3 shows the temperature dependence of the magnetization $M$ for $\mathrm{Cr}_{0.06} \mathrm{Ti}_{0.94} \mathrm{O}_{2-\delta}$ film measured from 2 to $400 \mathrm{~K}$ using SQUID with a magnetic field of 300 Oe applied in film plane. $M$ is constant up to $400 \mathrm{~K}$ except at very low temperature. The temperature dependence of the magnetization near $400 \mathrm{~K}$ suggests that there is no $\mathrm{CrO}_{2}$ impurity phase $\left(T_{C} \sim 398 \mathrm{~K}\right)$ in the films and confirms the XRD and TEM results.

In earlier investigations, the analogous series of oxides $\mathrm{Ti}_{n-2} \mathrm{Cr}_{2} \mathrm{O}_{2 n-1}(6 \leqslant n \leqslant 9)$ with triclinic structure ${ }^{6-9}$ were synthesized at high temperature. The corresponding $\mathrm{Cr} / \mathrm{Ti}$ ratio of the oxides is in the range of $2 / 7 \leqslant C r / T i \leqslant 1 / 2$. They are not ferromagnetic at RT. According to our results, $M$ decreases with increasing $\mathrm{Cr}$ content in the low $\mathrm{Cr}$ concentration region. This is consistent with earlier results on oxides with higher $\mathrm{Cr}$ content. It should be mentioned that, in the earlier investigations, the crystal structure of the high-

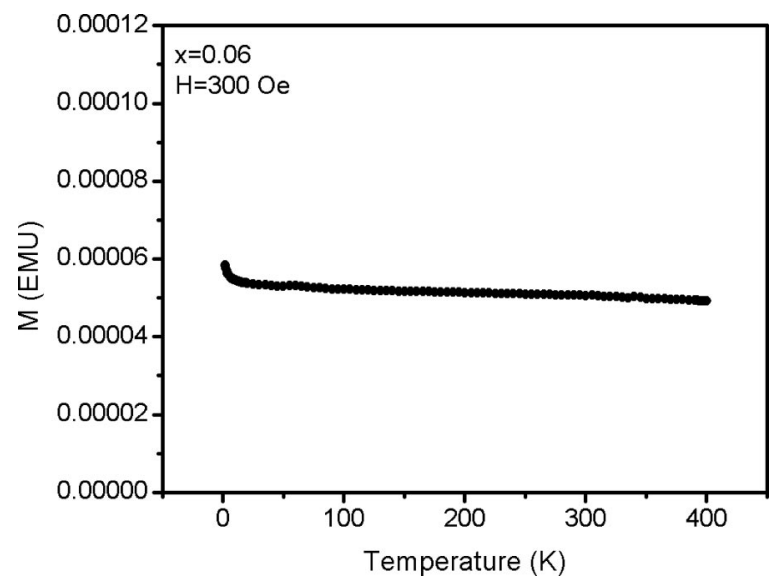

FIG. 3. Magnetization as a function of temperature for the $\mathrm{Cr}_{0.06} \mathrm{Ti}_{0.94} \mathrm{O}_{2-\delta}$ film measured from 2 to $400 \mathrm{~K}$ in a field of 300 Oe. The Curie temperature is above $400 \mathrm{~K}$.

temperature-synthesized oxides was not triclinic for $n<6$ or $n>9(\mathrm{Cr} / \mathrm{Ti}<2 / 7$ or $\mathrm{Cr} / \mathrm{Ti}>1 / 2) .{ }^{6-9}$ On the other hand, the structure is triclinic for our films prepared by PLD even though $\mathrm{Cr} / \mathrm{Ti}<2 / 7$.

Figure 4 compares the transport properties of the undoped reduced-rutile $\mathrm{TiO}_{2}$ and $\mathrm{Cr}_{x} \mathrm{Ti}_{1-x} \mathrm{O}_{2-\delta}(x=0.06$, $0.08,0.12,0.14)$ films. The films show increasing resistivity with decreasing temperature. All films exhibit nearly metallic conductance at RT and semiconducting behavior at lower temperatures. The resistivity at $300 \mathrm{~K}$ for $x=0.06$ is 0.0025 $\Omega \mathrm{cm}$, which is lower than that of the undoped film $(0.004$ $\Omega \mathrm{cm}$ ). The resistivity increases with increasing $\mathrm{Cr}$ concentration. In addition, $\mathrm{Cr}$ doping also changes the shape of $R-T$ curves.

The mechanism of the RT ferromagnetism in our films is not understood. Park et al., who have investigated the electronic structure of $\mathrm{Co} / \mathrm{Mn} / \mathrm{Fe} / \mathrm{Ni}$-doped anatase $\mathrm{TiO}_{2}$ with the local-spin-density approximation, suggest the ferromagnetism in the metallic phase is accounted for by the doubleexchange-like mechanism. ${ }^{10}$ The carriers are expected to be $n$-type. Such a mechanism cannot explain the experimental results presented here because the carriers of our films are

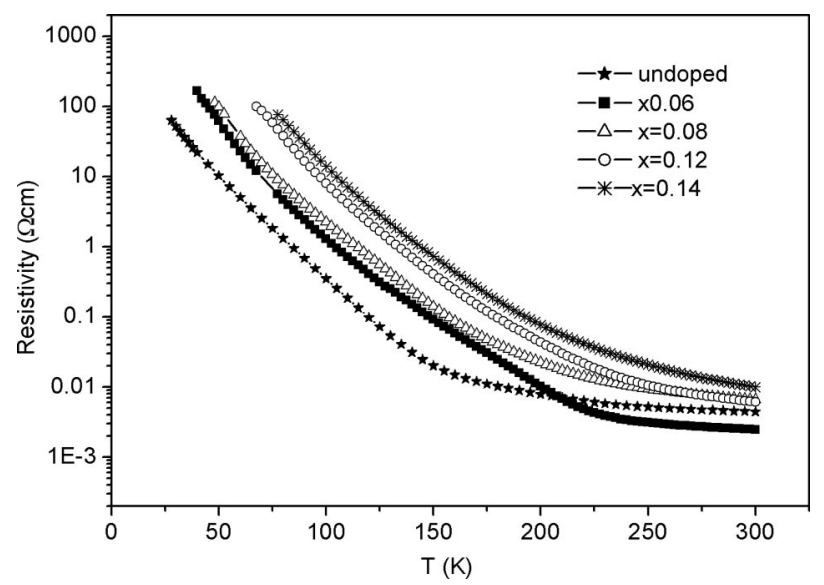

FIG. 4. Temperature dependence of the resistivity for the $\mathrm{Cr}_{x} \mathrm{Ti}_{1-x} \mathrm{O}_{2-\delta}$ ( $x=0.06,0.08,0.12,0.14)$ films. 
$p$-type. The origin of the magnetism should be related to the Zener-type, hole-mediated Ruderman-Kittel-KasuyaYosida interaction, which is responsible for the known magnetic semiconductor such as $(\mathrm{Ga}, \mathrm{Mn}) \mathrm{As} .{ }^{11}$ It turns out that, according to Dietl's model, holes are favored over electrons in order to achieve a ferromagnetic state at RT. The holes in our samples may play an important role in the coupling between the magnetic moments.

The highest magnetic moment of $2.9 \mu_{\mathrm{B}}$ per $\mathrm{Cr}$ atom found in our samples is larger than expected. Although it is possible that the entire contribution to the moment is from the $\mathrm{Cr}$ ions, it does not exclude the possibility that $\mathrm{Ti}$ moments are involved. When one pays attention to the unique structure of the reduced rutile, which produces $\mathrm{Ti}^{3+}$ ions with a $3 d$ moment, both $\mathrm{Cr}$ and $\mathrm{Ti}$ may contribute to the magnetization. This may lead to exchange couplings other than, or in addition to, the hole-mediated, Zener-type mechanism. The possible involvement of Ti $3 d$ electrons makes this system even more interesting and its understanding, possibly, more challenging.

So far, we have observed RT ferromagnetism in $\mathrm{Fe}$-, $\mathrm{Mn}$-, and $\mathrm{Cr}$-doped reduced-rutile films. $P$-type carriers have been found in all films suggesting the importance of the holes in the observed ferromagnetism. When grown under similar conditions, Co-doped $\mathrm{TiO}_{2}$ films contain nanoparticles of Co metal, and the films themselves are not ferromagnetic. The large magnetic moments for the Cr-doped films provide an opportunity for further exploring this interesting material.

\section{CONCLUSIONS}

The Cr-doped reduced-rutile $\mathrm{TiO}_{2}$ films are magnetic semiconductors up to $400 \mathrm{~K}$. The saturation magnetization of $\mathrm{Cr}_{x} \mathrm{Ti}_{1-x} \mathrm{O}_{2-\delta}$ films decreases with increasing $\mathrm{Cr}$ doping and the saturation magnetic moment of the $6 \% \mathrm{Cr}$-doped reduced films is $2.9 \mu_{\mathrm{B}}$ per $\mathrm{Cr}$ atom at RT. The large magnetic moments may originate from a number of sources. One such possibility is the involvement of the $3 d$ electrons of the $\mathrm{Ti}^{3+}$ ions. Another possibility is the formation of acceptor bound magnetic polarons, in which the spins of the holes and chromium are aligned via exchange interaction. The resistivity of the $\mathrm{Cr}_{x} \mathrm{Ti}_{1-x} \mathrm{O}_{2-\delta}$ films increases with increasing $\mathrm{Cr}$ concentration and with decreasing temperature.

\section{ACKNOWLEDGMENTS}

This work was supported by Sharp Laboratories of America and by Louisiana Board of Regents Support Fund Grant No. LEQSF(2000-03)-RD-B-10.

${ }^{1}$ G. a. Prinz, Science 282, 1660 (1998).

${ }^{2}$ H. Ohno, Science 281, 951 (1998).

${ }^{3}$ Z. Wang, W. Wang, J. Tang, L. D. Tung, L. Spinu, and W. Zhou, Appl. Phys. Lett. 83, 518 (2003).

${ }^{4}$ Z. Wang, J. Tang, L. D. Tung, W. Zhou, and L. Spinu, J. Appl. Phys. 93, 7870 (2003).

${ }^{5}$ S. B. Ogale, R. J. Chouhary, J. P. Buban, et al. Phys. Rev. Lett. 91(7), 077205 (2003).

${ }^{6}$ S. Kamiya, M. Yoshimura, and S. Somiya, Mater. Res. Bull. 15, 1303 (1980).

${ }^{7}$ H. Werner, Neues Jahrb. Mineral., Monatsh. 1974, 218 (1974).

${ }^{8}$ S. Andersson and L. Jahnberg, Ark. Kemi 21, 413 (1964).

${ }^{9}$ S. Andersson, A. Sundholm, and A. Magnell, Acta Chem. Scand. 13, 989 (1959).

${ }^{10}$ M. S. Park, S. K. Kwon, and B. I. Min, Phys. Rev. B 65, 161201 (2002).

${ }^{11}$ T. Dietl, H. Ohno, F. Matsukura, J. Cibert, and D. Ferrand, Science 287, 1019 (2000) 